\title{
Quantum Critical Magneto-transport at a Continuous Metal-Insulator Transition
}

\author{
P. Haldar, ${ }^{1, *}$ M. S. Laad, ${ }^{1, \dagger}$ S. R. Hassan,,${ }^{1}+$ Madhavi Chand, ${ }^{2,}$, and Pratap Raychaudhuri ${ }^{2}, \uparrow$ \\ ${ }^{1}$ Institute of Mathematical Sciences, Taramani, Chennai 600113, India and \\ Homi Bhabha National Institute Training School Complex, Anushakti Nagar, Mumbai 400085, India \\ ${ }^{2}$ Tata Institute of Fundamental Research, Homi Bhabha Rd, Colaba, Mumbai 400 005, India
}

(Dated: June 21, 2021)

\begin{abstract}
In contrast to the seminal weak localization prediction of a non-critical Hall constant $\left(R_{H}\right)$ at the Anderson metal-insulator transition (MIT), $R_{H}$ in quite a few real disordered systems exhibits both, a strong $T$-dependence and critical scaling near their MIT. Here, we investigate these issues in detail within a non-perturbative "strong localization" regime using cluster-dynamical mean field theory (CDMFT). We uncover $(i)$ clear and unconventional quantum-critical scaling of the GellMann-Law, or $\gamma$-function for magneto-transport, finding that $\gamma\left(g_{x y}\right)=\frac{d\left[\log \left(g_{x y}\right)\right]}{d[\log (T)]} \simeq \log \left(g_{x y}\right)$ over a wide range spanning the continuous MIT, very similar to that seen for the longitudinal conductivity, (ii) strongly $T$-dependent and clear quantum critical scaling in both transverse conductivity and $R_{H}$ at the MIT. We show that these surprising results are in comprehensive and very good accord with signatures of a novel Mott-like localization in NbN near the MIT, providing substantial support for our "strong" localization view.
\end{abstract}

At low temperatures $(T)$, transport in normal metals arises as a result of scattering of weakly interacting fermionic (Landau) quasiparticles amongst themselves, phonons and impurities [1]. Remarkably, it seems to hold even for $f$-electron systems, which are certainly strongly correlated Fermi liquids. However, this appealing quasiclassical description fails near metal-insulator transitions (MITs), where the Landau quasiparticle description itself breaks down [2]. In fact, in cuprates [3] and some $f$-electron systems [4], resistivity and Hall data can only be reconciled by postulating two distinct relaxation rates, arising from break-up of an electron, for the decay of longitudinal and transverse currents. In many cases, bad-metallic and linear-in- $T$ resistivities preclude use of Boltzmann transport views altogether, since the picture of weakly interacting Landau quasiparticles itself breaks down.

In disorder-driven MITs, resistivity and Hall effect have long been studied in the context of the seminal weak-localization (WL) theory [5]. These studies already threw up interesting hints regarding the inadequacy of WL approach upon attempts to reconcile criticality in (magneto)-transport [6]. Specifically, while both $\sigma_{x x}(n) \simeq\left(n_{c}-n\right)^{\nu}$ and $\sigma_{x y}(n) \simeq\left(n_{c}-n\right)^{\nu^{\prime}}$ turned out to be critical at the MIT, and the ratio $\nu^{\prime} / \nu \simeq 1$ in stark contrast to the value of 2 predicted at the Anderson MIT [7]. More recent work on intentionally disordered $\mathrm{NbN}$ [8], wherein the system is driven across $k_{F} l \simeq O(1)$ (here, $k_{F}$ is the Fermi wave-vector and $l$ the mean-free path, and $k_{F} l>>1$ describes a good metal, while $k_{F} l \leq 1.0$ describes a bad metal without welldefined electronic quasiparticles), shows clear signatures of an unusual type of localization at odds with theoretical predictions if one insists on an Anderson disorder-driven MIT: $(i) \rho_{x x}(T) \simeq C+A R_{H}(T)$, both increasing with reduction in $T$ over a wide range of $k_{F} l$ far before the MIT occurs (in $\mathrm{NbN}$, this is pre-empted by a superconductor- insulator transition (SIT) [8] at very low $T$ near the critical $\left(k_{F} l\right)_{c}$, and $(i i) \Delta R_{H} / R_{H} \simeq 0.69\left(\Delta \rho_{x x}(T) / \rho_{x x}\right)$, widely different from $\Delta R_{H} / R_{H} \simeq 2.0\left(\Delta \rho_{x x}(T) / \rho_{x x}\right)$ expected to hold in WL theory [9] $\left(k_{F} l>>1\right)$. These anomalies in both $\rho_{x x}$ and $R_{H}(T)$ are inexplicable within WL views (where $R_{H}$ is $T$-independent and non-critical at the MIT), and point toward a fundamentally new mechanism at work. Two possible reasons for this discord are: (1) electron-electron $(e-e)$ interactions grow [10] near a disorder-induced MIT and may destroy the oneelectron picture, and/or (2) such experiments maybe probing the "strong" localization regime of a disorder problem, where non-perturbative strong scattering effects may also destroy the one-electron picture. This is because Boltzmann approaches are untenable at the outset when $k_{F} l \simeq 1$, when a quasiparticle view itself breaks down.

Motivated by the above issues, we investigate magnetotransport near a continuous (at $T=0$ ) MIT. We choose the Falicov-Kimball model because $(i)$ it is the simplest model of correlated fermions exhibiting a continuous MIT, (ii) is exactly soluble within (cluster) dynamical mean-field theory ((C)DMFT) for arbitrarily strong interaction, and (iii) a two-site cluster-DMFT treats the all-important short-range correlations precisely on the length scale of $l \simeq k_{F}^{-1}$. Moreover, it is isomorphic to the binary-alloy Anderson disorder model, except that the FKM has annealed instead of quenched disorder. The Hamiltonian is

$H_{F K M}=-t \sum_{<i, j>}\left(c_{i}^{\dagger} c_{j}+h . c\right)+U \sum_{i} n_{i, d} n_{i, c}+\mu \sum_{i} n_{i, c}$

on a Bethe lattice with a semicircular band density of states (DOS) as an approximation to a $D=3$ lattice. $c_{i}\left(c_{i}^{\dagger}\right), d_{i}\left(d_{i}^{\dagger}\right)$ are fermion operators in dispersive 


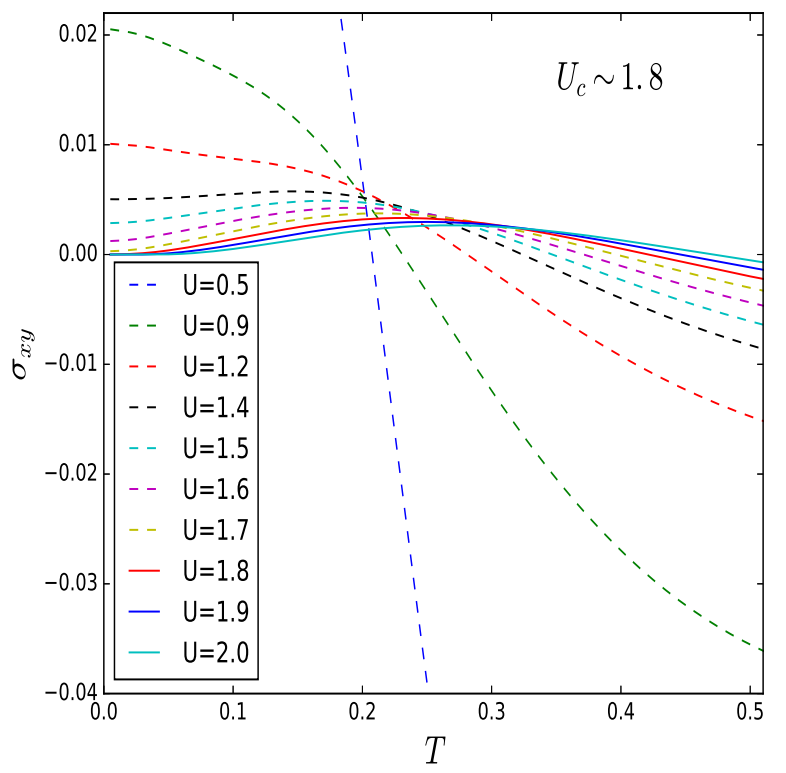

Figure 1. (Color online) Hall Conductivity $\left(\sigma_{x y}\right)$ as a function of temperature $(\mathrm{T})$ for different $\mathrm{U}$

band $(c)$ and dispersion less $(d)$ states, $t$ is the oneelectron hopping integral and $U$ is the onsite repulsion for a site-local doubly occupied configuration. Since $n_{i, d}=0,1, v_{i}=U n_{i, d}$ is also viewed as a static but annealed "disorder" potential for the $c$-fermions. We take non-interacting $c$-fermions half-bandwidth as unity i.e. $2 t=1$.

As studied earlier for the dc resistivity [11], we now use the exact-to- $O(1 / D)$ cluster propagators $G_{\mathbf{K}}(\omega)$ for each of the 2 -site cluster momenta $\mathbf{K}=(0,0),(\pi, \pi)$ to compute the full conductivity tensor, $\sigma_{a b}(T)$, with $a, b=x, y$. We neglect the vertex corrections to the Bethe-Salpeter equation (BSE) for all the intra-cluster momenta since they are negligible even within CDMFT, as can be seen, for example, within a cluster-to-orbital mapping [12, 13]. Thus, this constitutes an excellent approximation for computation of transport coefficients. Explicitly, the dc conductivity reads

$\sigma_{x x}(T)=\sigma_{0} \sum_{\mathbf{K}} \int_{-\infty}^{+\infty} d \epsilon v^{2}(\epsilon) \rho_{0}^{\mathbf{K}}(\epsilon) \int_{-\infty}^{+\infty} d \omega A_{\mathbf{K}}^{2}(\epsilon, \omega)\left(\frac{-d f}{d \omega}\right)$

where $\sigma_{0}=\frac{\pi e^{2}}{\hbar D a} \simeq\left(10^{-3}-10^{-2}\right)(2 / D)(\mu \Omega) \cdot \mathrm{cm}-1$, $\rho_{0}^{\mathbf{K}}(E)$ the "partial" unperturbed DOS used in earlier work [14] and $A_{\mathbf{K}}(E)$ the intra-cluster CDMFT one-fermion spectral function. The Hall conductivity is a more delicate quantity to compute [15]. Fortunately,absence of vertex corrections comes to the rescue and we find
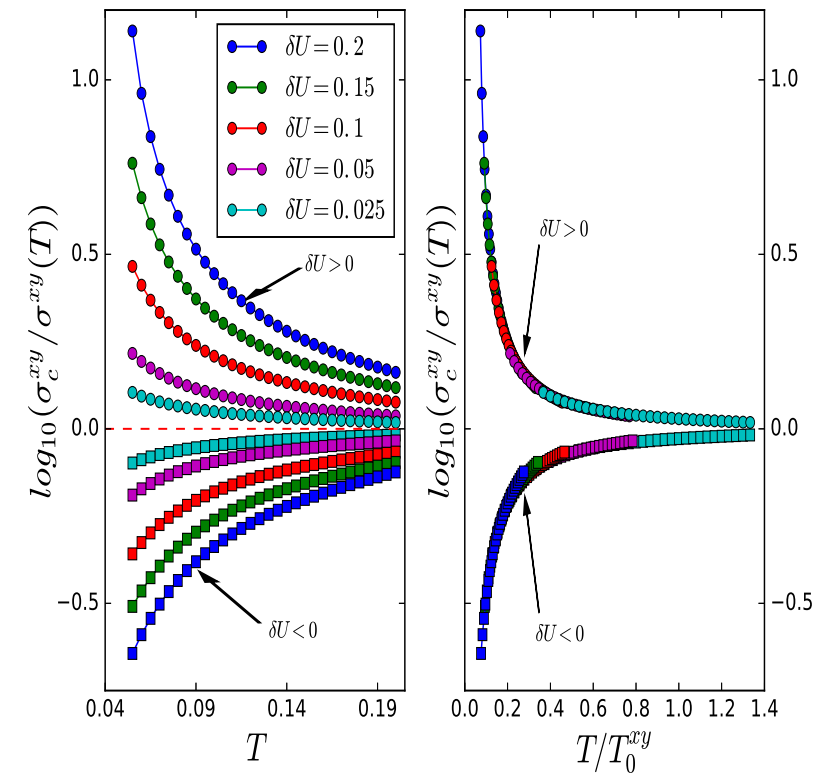

Figure 2. (Color online)(a) In left panel, $\log _{10}\left(\frac{\sigma_{c}^{x y}}{\sigma^{x y}(T)}\right)$ as a function of temperature $\mathrm{T}$ for $\delta U= \pm 0.025,0.05,0.1,0.15,0.2 ; \sigma_{c}^{x y}$ is the "separatrix". (b)In right panel, scaling the data along T-axis by scaled temperature $T_{0}^{x y}$.

$\sigma_{x y}(T)=\sigma_{x y, 0} B \sum_{\mathbf{K}} \int d \epsilon v^{2}(\epsilon) \rho_{0}^{\mathbf{K}}(\epsilon) \epsilon \int d \omega A_{\mathbf{K}}^{3}(\epsilon, \omega)\left(\frac{d f}{d \omega}\right)$

with $\sigma_{x y, 0}=-\frac{2 \pi^{2}|e|^{3} a}{3 \hbar^{2}}\left(1 / 2 D^{2}\right)$, and $B$ the magnetic field. Now, the Hall constant is simply $R_{H}(T)=\frac{\sigma_{x y}}{B \sigma_{x x}^{2}}$ and the Hall angle is $\cot \theta_{H}=\frac{\sigma_{x x}}{\sigma_{x y}}$. we show the offdiagonal conductivity, $\sigma_{x y}(U, T)$ as a function of $U$ from small- to large $U$ across the continuous MIT occurring at $U_{c}=1.8$ [14]. First, we show results for the temperaturedependent off-diagonal conductivity, $\sigma_{x y}(T)$ as a function of $U$ across the continuous Mott transition.

We use Equation 3 to compute $\sigma_{x y}(T, U)$. In Fig. 1 we show $\sigma_{x y}(T, U)$ as a function of temperature (T) for different disorder values (U). A clear change of slope at low $T<0.05 t$ occurs around $U \simeq 1.3$, which seems to correlate with the bad-metal-to-bad-insulator crossover in the $d c$ resistivity in our earlier study [11]. Close to the MIT, $\rho_{d c}(T)$ diverges approximately like $\exp \left(E_{g} / k_{B} T\right)$ as $T \rightarrow 0$ in this regime, $R_{H}(T \rightarrow 0)$ diverges as it must, since the MIT is accompanied by loss of carriers due to gap opening. A clear change of slope (for $T<0.05 t$ ) occurs around $U=1.3$, and $\sigma_{x y}(T) \simeq T^{1.2}$ around $U_{c}$. The $d c$ resistivity $\rho_{x x}(T)$ shows extremely bad-metallic behaviour at lowest $T$, beautiful mirror symmetry and novel "Mott-like" scaling [11] precisely in this regime. It is obviously of interest to inquire whether the novel 


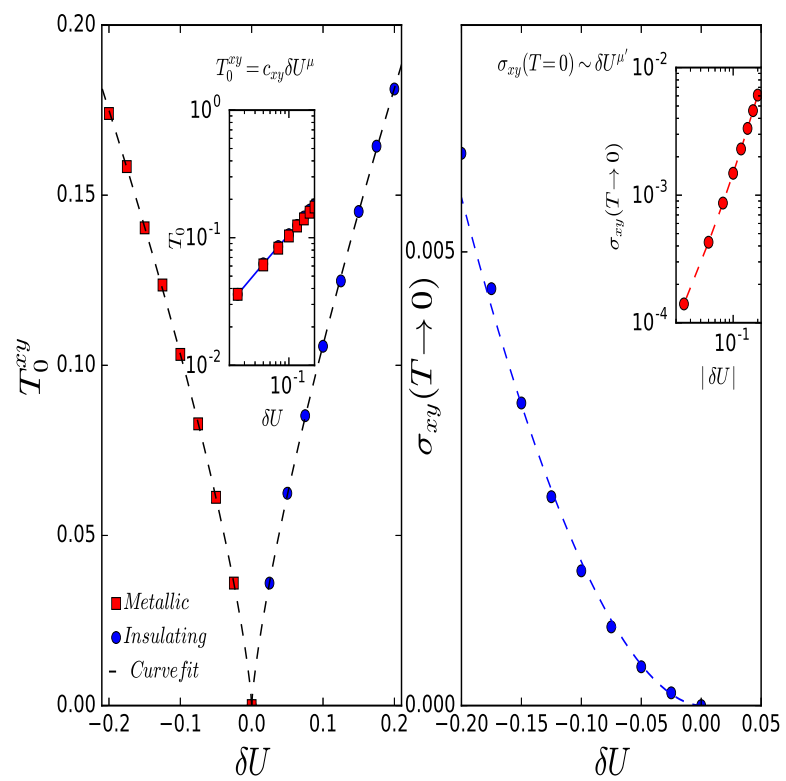

Figure 3. (Color online) (a) In left panel, scaling parameter $T_{0}^{x y}$ as a function of control parameter $\delta U=U-U_{c}$; the inset illustrates power law dependence of scaling parameter $T_{0}^{x y}=c|\delta U|^{\mu}$. (b)In right panel, $\sigma_{x y}(T \rightarrow 0)$ as a function of control parameter $\delta U=U-U_{c}$; the inset illustrates power law dependence of $\sigma_{x y}(T \rightarrow 0)=c|\delta U|^{\mu^{\prime}}$.

features seen in $\rho_{x x}(U, T)$ are also reflected in magnetotransport near the "Mott" QCP. To facilitate this possibility, we show $\log _{10}\left(\frac{\sigma_{c}^{x y}}{\sigma^{x y}(T)}\right)$ versus $T$ in the left panel of Fig.2, finding that the family of $1 / \sigma^{x y}(U, T)$ curves also exhibit a near-perfect "mirror" symmetry over an extended region around $1 / \sigma_{(c)}^{x y}(U, T)$, strongly presaging quantum critical behaviour. To unearth this feature, we also show $\log _{10}\left(\frac{\sigma_{c}^{x y}}{\sigma^{x y}(T)}\right)$ versus $T / T_{0}^{x y}$ in the right panel of Fig.2, where we have repeated the unbiased method of introducing a $T_{0}^{x y}(U)$ to rescale all metallic and insulating curves on to two universal curves. Remarkably, as for the $\rho_{x x}$-scaling, we find, as shown in the left panel of Fig.3, that $T_{0}^{x y}$ vanishes precisely at the MIT. Clear scaling behaviour we find testifies to a remarkable fact: the novel scaling features found earlier in $d c$ resistivity are also clearly manifest in the off-diagonal resistivity.

Even clearer characterization of the quantum critical features obtains when we compute the $\gamma$-function [7] (this is the analogue of the well-known $\beta$-function for the longitudinal conductivity) for $\sigma_{x y}(U, T)$, defined by $\gamma\left(g_{x y}\right)=\frac{d\left[\ln \left(g_{x y}\right)\right]}{d[\ln (T)]}$, with $g_{x y}=\sigma^{x y}(T) / \sigma_{c}^{x y}$. As shown in Fig.4, it is indeed remarkable that it clearly varies as $\ln \left(g_{x y}\right)$, and is continuous through $\delta U=0$. This shows that it has precisely the same form on both sides of the MIT, which is exactly the feature needed for genuine quantum criticality. These features resemble those

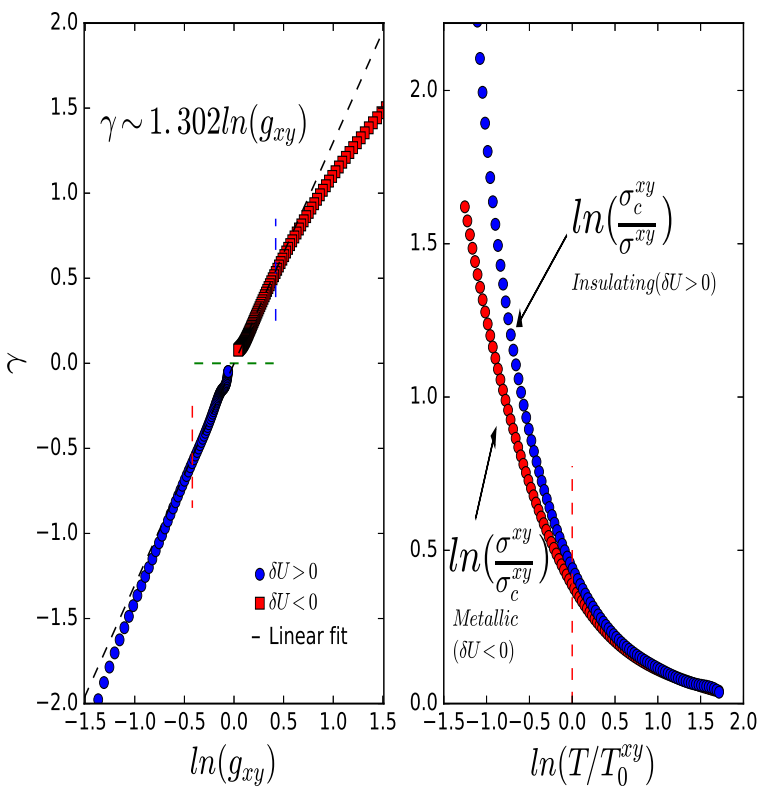

Figure 4. (Color online)(a) In left panel, $\gamma$-function shows linear in $\ln \left(g_{x y}\right)$ behaviour close to the transition. Squares are for metallic branch $(\delta U<0)$ and circles ones are for insulating branch $(\delta U>0)$; vertical dashed indicate the region where mirror symmetry of curved is found. (b) In right panel, reflection symmetry of scaled curved close to the transition.

found for QC scaling in $\rho_{x x}[11]$, showing that, like $\beta(g)$, $\gamma\left(g_{x y}\right) \simeq \ln \left(g_{x y}\right)$ deep into the metallic phase. Thus, we have found that the full $d c$ conductivity tensor reflects the strong coupling nature of the "Mott" QCP, attesting to its underlying non-perturbative origin in Mott-like (strong scattering) physics.

That $\gamma\left(g_{x y}\right) \simeq \ln g_{x y}$ holds on both sides of the MIT implies that its two branches must display "mirror symmetry" over an extended range of $g_{x y}$. In Fig.4, left panel, we indeed see that magneto-transport around the QCP exhibits well-developed reflection symmetry (bounded by dashed vertical lines), It is also manifest in the right panel of Fig.4, where $\sigma_{c}^{x y} / \sigma^{x y}(\delta U)=\sigma^{x y}(-\delta U) / \sigma_{c}^{x y} ;$ i.e, they are mapped onto each other under reflection around $U_{c}$, precisely as found earlier for the $d c$ resistivity. As a final check, we also show (see Fig. 5) that $\log \left(\sigma_{c}^{x y} / \sigma^{x y}(T)\right.$ is a universal function of the "scaling variable" $\delta U / T^{1 / \mu}$. Thus, our study explicitly shows the novel quantum criticality in magneto-transport at the "Mott" QCP (associated with a continuous Mott transition) in the FKM at strong coupling.

In an Anderson model framework, scaling of $\sigma_{x y}$ is long known [7]. Our findings are very distinct from expectations for an Anderson-like transition: observe that we find $T_{0}^{x y}(\delta U) \simeq c_{x y}|\delta U|^{\mu}$ (in left panel of Fig. 3) with $\mu \simeq 0.75=3 / 4$ (in inset) on both sides of $U_{c}$, as re- 


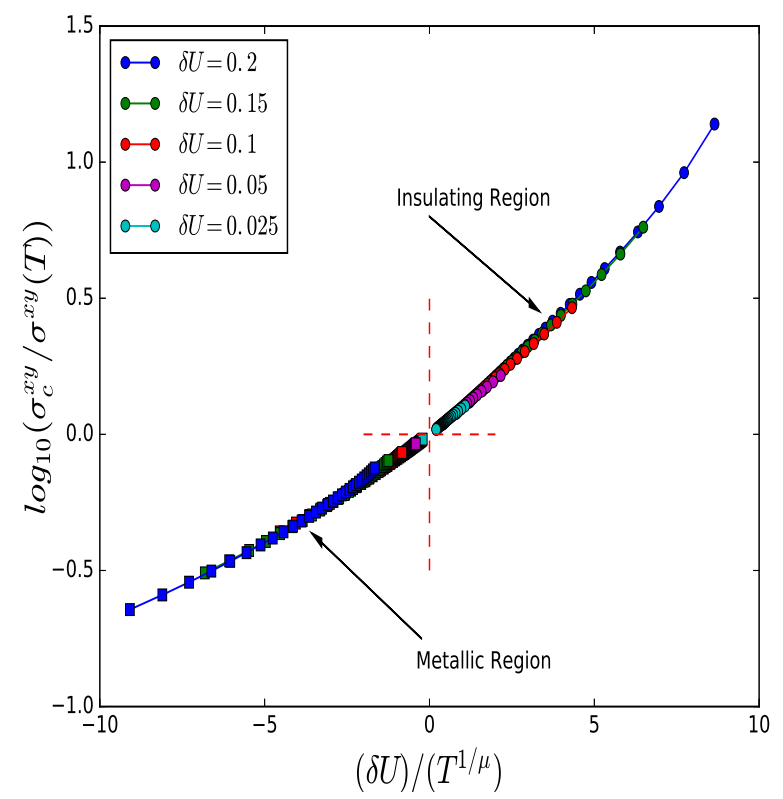

Figure 5. (Color online) $\log _{10}\left(\frac{\sigma_{c}^{x y}}{\sigma^{x y}(T)}\right)$ vs $(\delta U) / T^{\frac{1}{\mu}}$, where $\delta U=U-U_{c}$.

quired for genuinely quantum critical behaviour. This strongly contrasts with the $T_{0}^{x x}(\delta U) \simeq c|\delta U|^{z \nu}$ with $z \nu=1.32 \simeq 4 / 3$ found for the $d c$ resistivity [11]. Further, in the right panel of Fig.3, we also show that $\sigma_{x y}=\sigma_{0, x y}\left(U_{c}-U\right)^{\mu^{\prime}}$ with $\mu^{\prime}=1.8$ (in inset), quite distinct from $\nu \simeq 4 / 3$ found earlier for $\sigma_{x x}(U)$.

Along with our finding of $\sigma_{x x}(T) \simeq T$ and $\sigma_{x y}(T) \simeq$ $T^{1.2}$ at the MIT, these findings have very interesting consequences: $(i)$ the Hall constant is critical at the MIT. We find $R_{H}^{-1} \simeq \sigma_{x x}^{2} / \sigma_{x y} \simeq\left(U_{c}-U\right)^{0.8}$, whereas $R_{H}$ is non-critical [7] at the Anderson MIT, (ii) $R_{H}$ is also strongly $T$-dependent and divergent at the MIT, varying like $R_{H}(T) \simeq T^{-0.8}$, whereas $R_{H} \simeq(n e c)^{-1}$ in an Anderson disorder model. Concomitantly, the Hall angle also exhibits anomalous behaviour: (iii) $\tan \theta_{H}(T) \simeq T^{0.2}$ and $\tan \theta_{H}(U) \simeq\left(U_{c}-U\right)^{1 / 2}$ in the quantum critical region. Our results are distinct from expectations from a Landau FL and Anderson-MIT views. At an Anderson MIT [7], $R_{H}=(n e c)^{-1}$ is $T$-independent and noncritical at the MIT. In the metallic phase, use of semiclassical ideas dictates that both $\beta(g)$ and $\gamma\left(g_{x y}\right)$ scale like $(d-2)-A / g$, and the quantum correction to the Hall conductance is twice as big as for the Ohmic conductance. The stringent assumption under which this holds is that the inverse Hall constant (related to $h(L)=L^{d-2} / R_{H} B$ in Abrahams et al.) scales classically like $h(L) \simeq L^{d-2}$ for small $B$ (large $h$ ). It is precisely this assumption that breaks down when one considers the Mott MIT, where $R_{H}$ is critical at the MIT (see above). They are also different from expectations in a correlated LFL: a strongly $T$-dependent $R_{H}$ close to a Mott MIT in a Hub-

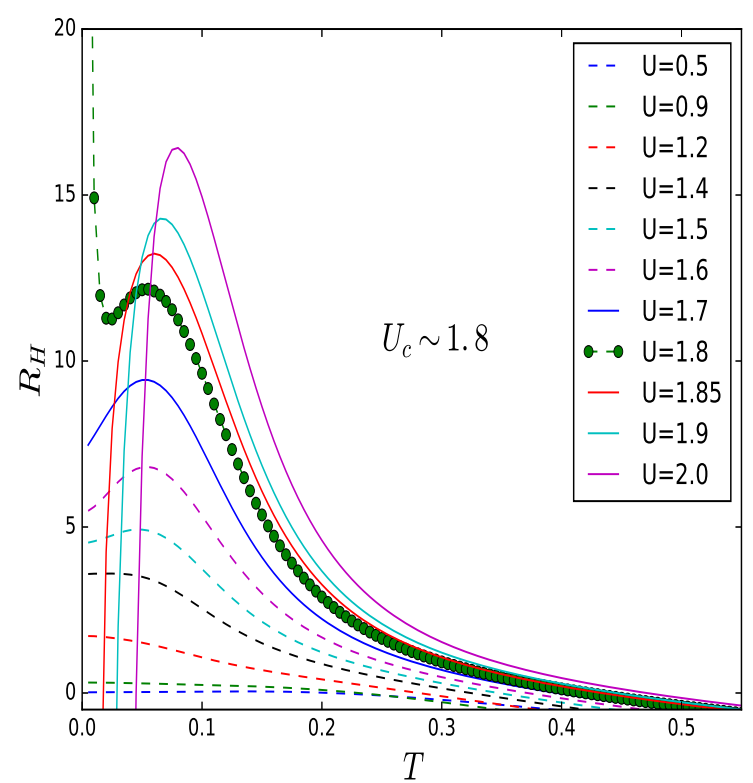

Figure 6. (Color online) Hall co-efficient $R_{H}$ as a function of temperature $\mathrm{T}$ for different $\mathrm{U}$.

bard model framework is long known [16]. However, in a DMFT framework, $R_{H}(T)$ exhibits a recovery of correlated Landau-Fermi liquid behaviour below a low- $T$ lattice coherence scale. Moreover, the MIT there is a firstorder transition. In the FKM, the metallic state remains bad-metallic and incoherent down to lowest $T$, and the MIT is continuous. The Mott-like character of the associated QCP is revealed by the observation of $\beta(g) \simeq$ $\log (g)$ and $\gamma\left(g_{x y}\right) \simeq \log \left(g_{x y}\right)$.

In Fig.6, we show $R_{H}(U, T)$ versus Temperature (T). Both are indeed markedly $T$-dependent. For an Anderson MIT, $R_{H}$ would be non-critical. In a LFL metal, one expects $\sigma_{x x}(T)=1 / \rho_{d c}(T)=A T^{2}$, while $\sigma_{x y}(T) \simeq T^{-4}$ at low $T$. In that case, we end up with a $T$-independent $R_{H}$ and $\cot \theta_{H}(T)=c T^{2}$. This is the expected behaviour for a LFL, where a single relaxation rate governs the $T$-dependent relaxation of longitudinal and Hall currents. Very different $T$-dependences we find here testify to the breakdown of this intimate link between LFL quasi-particles and this conventional behaviour, and that the results we find are direct consequences of the destruction of LFL quasi-particles at strong coupling. They render semiclassical Boltzmann arguments (based on validity of $k_{F} l>>1$ ) inapplicable at the outset.

We now turn to experiments to investigate how our theory stands this stringent test. Recent work on $\mathrm{NbN}$ [8] most clearly reveals ill-understood signatures of localization incompatible with weak localization predictions. In $\mathrm{NbN}$, the effect of intentional charge disorder is to cause a random variation in the local atomic potential, which increases as $k_{F} l$ is reduced by increasing the dis- 

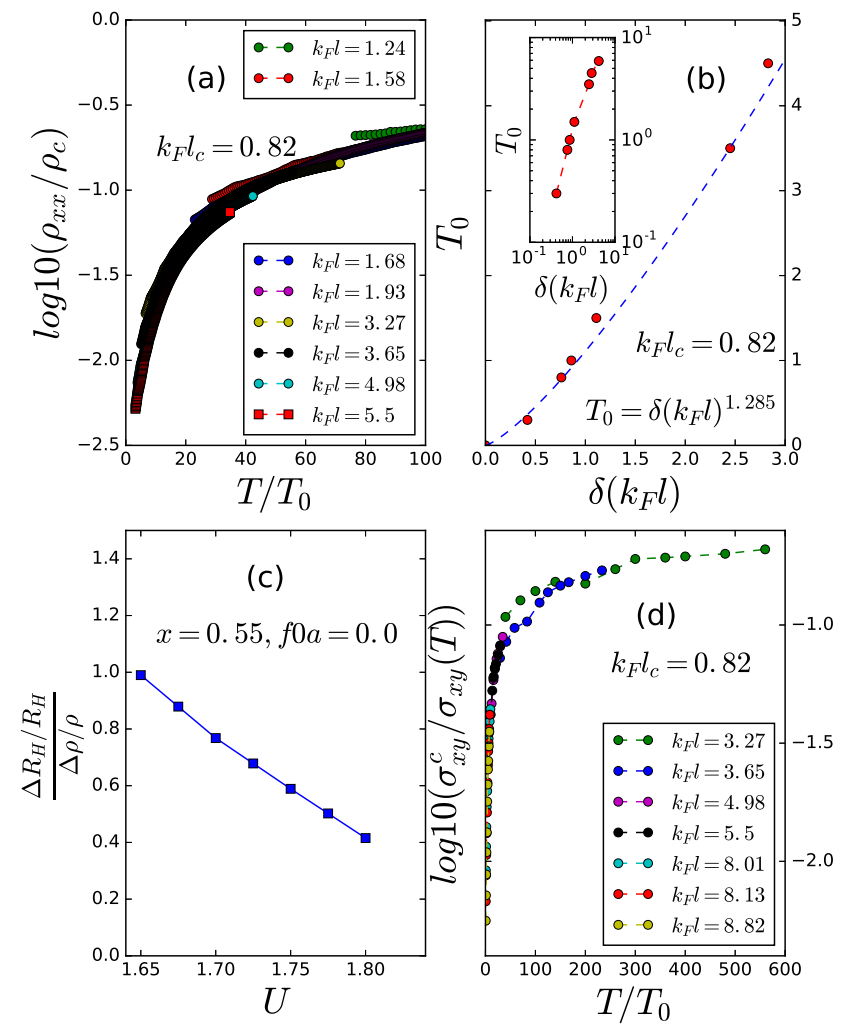

Figure 7. (Color online) (a). Resistivity data from Chand et al. [8], replotted as $\log \left(\rho_{x x}(T) / \rho_{c}\right)$ versus $T / T_{0}$ with $T_{0}\left(\delta k_{F} l\right) \simeq\left|\delta k_{F} l\right|^{1.3}$ in Panel $(\mathrm{b})$, in excellent accord with theory [11]. In Panel (c), we show that the theoretical ratio $\frac{\Delta R_{H} / R_{H}}{\Delta \rho / \rho}$ is in the range of $0.5-0.7$ near the Mott QCP, again in good qualitative accord with the value of 0.69 from Hall data [8]. In Panel (d), we show clear scaling of the experimentally extracted $\log \left(\sigma_{x y}^{(c)} / \sigma_{x y}(T)\right)$ in very good accord with theory for the same sample set used for Panel (a). The $\sigma_{x y}(T)$ is constructed from the experimental dc resistivity and Hall constant $\left(R_{H}\right)$. The $R_{H}$ at the critical $k_{F} l$ is calculated from extrapolation of the experimental Hall constant $\left(R_{H}\right)$ down to $\left(k_{F} l\right)_{c}=0.82$, as shown in Fig. 8

order level. Following Freericks et al. [17], we posit that the FKM is a suitable effective model for materials like $\mathrm{Ta}_{x} \mathrm{~N}$ and $N b_{x} N$, where carriers interact locally with randomly distributed charge disorder. We have reanalyzed Chand et al.'s data on $\mathrm{NbN}$ in light of the above results to test how our strong coupling view performs relative to data. To make meaningful contact with data on $\mathrm{NbN}$, we make a reasonable assumption that increasing $U / t$ in the FKM corresponds to decreasing $k_{F} l$, since the scattering strength should increase with $U / t$, reducing $k_{F} l$ to $O(1)$ [8] near the MIT. We find: as shown in Fig. 7(a), that $(i) \log \left(\rho_{x x}(T) / \rho_{c}\right)$ on the (bad) metallic side scales with $T / T_{0}\left(k_{F} l\right)$ exactly as predicted by our
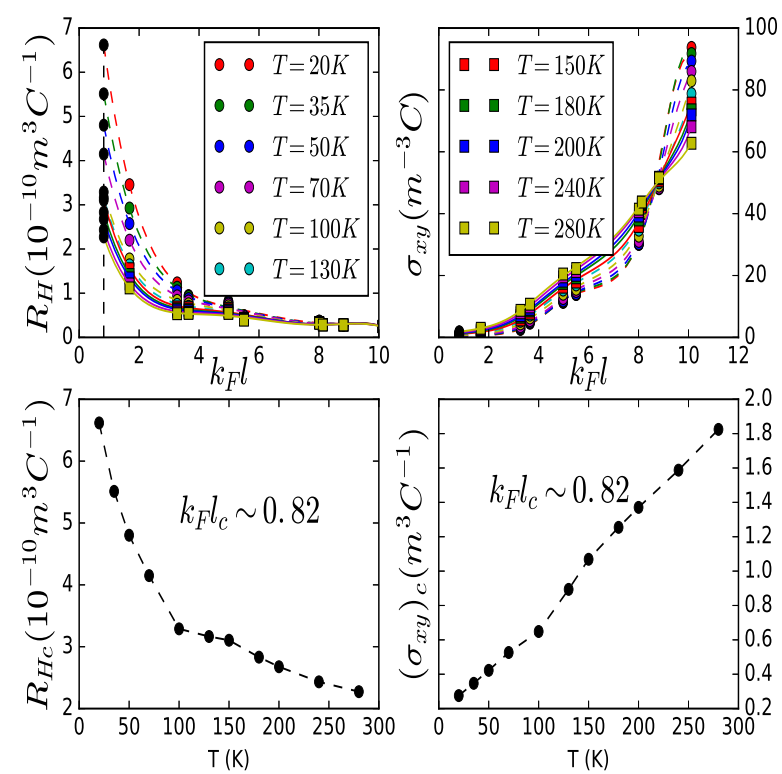

Figure 8. (Color online)(a) In top-left panel, Hall constant $R_{H}$ as a function of $k_{F} l$ for various temperature T, black circles are the values of $R_{H}$ after extrapolating the curves upto critical $k_{F} l=0.82$ (b) In top-right panel, Hall conductivity $\sigma_{x y}$ calculated from the Hall constant $\left(R_{H}\right)$ and dc resistivity $\left(\rho_{x x}\right)$ as a function of $k_{F} l$ for various temperature $\mathrm{T}$ (c) In bottom-left panel, Hall constant $R_{H}$ as a function temperature $(\mathrm{T})$ at the critical $k_{F} l=0.82$ calculated from the extrapolation, (d) In bottom-right panel, Hall conductivity $\sigma_{x y}$ as a function temperature $(\mathrm{T})$ at the critical $k_{F} l=0.82$

theory [11]. Further, the data analysis shows (Fig. 7(b)) that $T_{0}\left(k_{F} l\right) \simeq\left(k_{f} l-\left(k_{f} l\right)_{c}\right)^{z \nu}$ with $z \nu \simeq 1.3$, again in excellent accord with theory if we identify decreasing $k_{F} l$ with increasing $U$ in our model. (ii) interestingly, our $\rho_{x x}(T), R_{H}(T)$ results reproduce the detailed $T$-dependence seen in data [8] with only one adjustable parameter $(U)$. (iii) even more remarkably, we find that $\left(\Delta R_{H} / R_{H}\right) /\left(\Delta \rho_{x x} / \rho_{x x}\right.$, shown in Fig. $7(\mathrm{c})$, achieves values between 0.5 and 0.7 close to the MIT (between $1.5 \leq U \leq 1.9)$ in our model, in very good accord with 0.69 extracted in experiment. Finally, in Fig. $7(\mathrm{~d})$, we uncover quantum critical scaling in $1 / \sigma_{x y}(T)$ as a function of $k_{F} l$ from data on $\mathrm{NbN}$, which is expected in our model, since both $\sigma_{x x}, \sigma_{x y}$ exhibit such novel scaling behaviour. Since $R_{H}$ is difficult to extract reliably in very bad-metallic samples (with $k_{F} l<3.0$ ) close to the MIT, we resorted to a careful extrapolation of the Hall conductivity $\left(\sigma_{x y}\right)$ and Hall constant $\left(R_{H}\right)$ to smaller values of $k_{F} l$.

In Fig. 8, we show the results of a careful fitting of the experimental data down to $k_{F} l \simeq O(1)$ (in fact, the critical $\left(k_{F} l\right)_{c}$ is now consistent with 0.82 , which is the critical value for the longitudinal dc conductivity). Using these extrapolated fits to the dc conductivity tensor as 
a function of $k_{F} l$, we constructed Fig. $7(\mathrm{~d})$ in the main text. This makes our analysis consistent with a single $\left(k_{F} l\right)_{c} \simeq 0.82$ for both $\sigma_{x x}\left(k_{F} l, T\right)$ and $\sigma_{x y}\left(k_{F} l, T\right)$.

Taken together, earlier results of Chand et al. [8], now suitably reanalyzed in light of our CDMFT results, receive comprehensive explication within a "strong localization" view adopted here, lending substantial support to the view that the novel findings in $\mathrm{NbN}$ are representative of strong scattering effects near a continuous MIT, and involve microscopic processes beyond perturbativein- $\left(1 / k_{F} l\right)$ approaches.

Thus, to conclude, we have presented clear evidence of novel quantum critical behaviour in magneto-transport near a continuous MIT by a careful scaling analysis of CDMFT results for the off-diagonal conductivity for the FKM in the strong localization limit. We find that the loss of the quasiparticle pole structure at strong coupling $\left(k_{F} l \simeq 1\right)$ leads to a rather distinct "Mott"-like quantum criticality, necessitating substantial modification of the quasiclassical Drude-Boltzmann transport schemes to study (magneto)-transport. The resulting quantum criticality we find is closer to that expected from the opposite limit of strong localization based on a real-space locator expansion [18, 19], as manifested in $\gamma\left(g_{x y}\right) \simeq \ln \left(g_{x y}\right)$. Comprehensive and very good explication of recent data on $\mathrm{NbN}$ lend substantial experimental support to this Mott-like view. We suggest that strongly disordered electronic systems that show a bad-metallic resistivity and sizeable $T$-dependent Hall constant would be promising candidates to unearth such novel quantum-critical magneto-transport at a continuous MIT. Finally, the similarity of QC scaling in resistivity in earlier work [11] to the Mott QC scaling in the Hubbard model [20] above the finite- $T$ critical end-point suggests that related features discussed above may also manifest in wider classes of strongly correlated Mott materials.

* prosenjit@imsc.res.in

$\dagger$ mslaad@imsc.res.in

¥ shassan@imsc.res.in

$\S$ madhavichand2009@gmail.com

ฯ praychaudhuri2014@gmail.com

[1] D. Pines and P. Nozieres, "Theory of quantum liquids," Perseus Books, Cambridge (Mass) (1999).

[2] Masatoshi Imada, Atsushi Fujimori, and Yoshinori Tokura, "Metal-insulator transitions," Rev. Mod. Phys. 70, 1039-1263 (1998).

[3] T. R. Chien, Z. Z. Wang, and N. P. Ong, "Effect of zn impurities on the normal-state hall angle in single-crystal $\mathrm{yba}_{2} \mathrm{Cu}_{3-x} \mathrm{Zn}_{x} \mathrm{O}_{7-\delta}$," Phys. Rev. Lett. 67, 2088-2091 (1991).

[4] S. Paschen, T. Lühmann, S. Wirth, P. Gegenwart,
O. Trovarelli, C. Geibel, F. Steglich, P. Coleman, and Q. Si, "Hall-effect evolution across a heavy-fermion quantum critical point," Nature 432, 881-885 (2004).

[5] Patrick A. Lee and T. V. Ramakrishnan, "Disordered electronic systems," Rev. Mod. Phys. 57, 287-337 (1985).

[6] Stuart B. Field and T. F. Rosenbaum, "Critical behavior of the hall conductivity at the metal-insulator transition," Phys. Rev. Lett. 55, 522-524 (1985).

[7] Boris Shapiro and Elihu Abrahams, "Scaling theory of the hall effect in disordered electronic systems," Phys. Rev. B 24, 4025-4030 (1981).

[8] Madhavi Chand, Archana Mishra, Y. M. Xiong, Anand Kamlapure, S. P. Chockalingam, John Jesudasan, Vivas Bagwe, Mintu Mondal, P. W. Adams, Vikram Tripathi, and Pratap Raychaudhuri, "Temperature dependence of resistivity and hall coefficient in strongly disordered nbn thin films," Phys. Rev. B 80, 134514 (2009).

[9] B. L. Altshuler, D. Khmel'nitzkii, A. I. Larkin, and P. A. Lee, "Magnetoresistance and hall effect in a disordered two-dimensional electron gas," Phys. Rev. B 22, 5142-5153 (1980).

[10] Alexander Punnoose and Alexander M. Finkel'stein, "Metal-insulator transition in disordered two-dimensional electron systems," Science 310, 289-291 (2005).

[11] P. Haldar, M. S. Laad, and S. R. Hassan, "Quantum critical transport at a continuous metal-insulator transition," Phys. Rev. B 94, 081115 (2016).

[12] Jan M. Tomczak and Silke Biermann, "Optical properties of correlated materials: Generalized peierls approach and its application to vo2," Phys. Rev. B 80, 085117 (2009).

[13] K. Haule and G. Kotliar, "Optical conductivity and kinetic energy of the superconducting state: A cluster dynamical mean field study," EPL (Europhysics Letters) 77, 27007 (2007).

[14] P. Haldar, M. S. Laad, and S. R. Hassan, "Realspace cluster dynamical mean-field approach to the Falicov-Kimball model: An alloy-analogy approach," Phys. Rev. B 95, 125116 (2017).

[15] Ekkehard Lange, "Memory-function approach to the hall constant in strongly correlated electron systems," Phys. Rev. B 55, 3907-3928 (1997).

[16] Ekkehard Lange and Gabriel Kotliar, "Magnetotransport in the doped mott insulator," Phys. Rev. B 59, 1800-1807 (1999).

[17] J. K. Freericks and V. Zlatić, "Exact dynamical mean-field theory of the falicov-kimball model," Rev. Mod. Phys. 75, 1333-1382 (2003).

[18] P. W. Anderson, "Absence of diffusion in certain random lattices," Phys. Rev. 109, 1492-1505 (1958).

[19] V. Dobrosavljević, Elihu Abrahams, E. Miranda, and Sudip Chakravarty, "Scaling theory of two-dimensional metal-insulator transitions," Phys. Rev. Lett. 79, 455-458 (1997).

[20] H. Terletska, J. Vučičević, D. Tanasković, and V. Dobrosavljević, "Quantum critical transport near the mott transition," Phys. Rev. Lett. 107, 026401 (2011). 